\title{
Ideação suicida na população universitária
}

\author{
Suicide ideation in the university population \\ Ideación Suicida em la población universitaria
}

\section{Resumo}

Analisar se há ideações suicidas, identificar quais são as motivações, e descrever o perfil sociodemográfico e econômico dos estudantes universitários. Estudo quantitativo, transversal, com graduandos da Universidade do Estado de Minas Gerais - UEMG - Divinópolis no ano de 2020. Utilizou-se Questionário Sociodemográfico, Suicide Ideation Questionnare (QIS), Escala de Beck e Escala de Satisfação com o Suporte Social (ESSS). Realizou-se o teste quiquadrado, exato de Fisher, cálculo da Odds-Ratio (OR). Ambos os testes estatísticos foram realizados por meio do programa Statistical Package for Social Sciences (SPSS). Participaram do estudo 130 universitários, com predomínio do sexo feminino (81,5\%), faixa etária entre 21 e 25 anos $(61,5 \%)$ e solteiros $(78,5 \%)$. Do total da amostra, 28 $(21,53 \%)$ apresentaram risco de ter ideação suicida. Foi elencado que os estudantes que, residem em outra cidade, possuem 1,52 vezes mais chances de apresentarem ideação suicida ( $\mathrm{IC}=0,65-3,55)$, que possuem renda mensal menor ou igual a um salário mínimo aumenta em 2 vezes as chances de ter ideação suicida (IC: 0,74-0,53) e que alegam sofrer de algum transtorno mental ou comportamental aumenta em 1,40 vezes as chances de ideação suicida (IC $=0,60$ 3,25). Conhecer as características mentais dos universitários, possibilita planejar intervenções conjuntas na comunidade acadêmica e desenvolver estratégias de prevenção e promoção diminuindo a ideação suicida nesse público.

Palavras-chave: Ideação suicida; Estudantes; Saúde do estudante; Universidades.

\begin{abstract}
Analyze whether there are suicidal ideations, identify what the motivations are, and describe the sociodemographic and economic profile of university students. Quantitative, cross-sectional study with undergraduate students from the State University of Minas Gerais - UEMG - Divinópolis in 2020. A Sociodemographic Questionnaire, Suicide Ideation Questionnare (QIS), Beck Scale and Satisfaction with Social Support Scale (ESSS) were used. The chisquare test, Fisher's exact test, calculation of the Odds-Ratio (OR) was performed. Both statistical tests were performed using the Statistical Package for Social Sciences (SPSS) program. 130 university students participated in the study, predominantly female (81.5\%), aged between 21 and 25 years $(61.5 \%)$ and single $(78.5 \%)$. Of the total sample, $28(21.53 \%)$ were at risk of having suicidal ideation. It was listed that students who live in another city are 1.52 times more likely to have suicidal ideation $(\mathrm{CI}=0.65-3.55)$, who have a monthly income less than or equal to the minimum wage increases by 2 times the chances of having suicidal ideation (CI: $0.74-0.53$ ) and claiming to suffer from some mental or behavioral disorder increases by 1.40 times the chances of suicidal ideation (CI=0.60 to 3.25 ). Knowing the mental characteristics of university students makes it possible to plan joint interventions in the academic community and develop prevention and promotion strategies, reducing suicidal ideation in this public.
\end{abstract}

Keywords: Suicidal ideation; Students; Student health; Universities. 


\begin{abstract}
Resumen
Analizar si existen ideas suicidas, identificar las motivaciones y describir el perfil sociodemográfico y económico de los estudiantes universitarios. Estudio cuantitativo, transversal con estudiantes de pregrado de la Universidad Estatal de Minas Gerais - UEMG - Divinópolis en 2020. Se utilizó Cuestionario Sociodemográfico, Cuestionario de Ideación Suicida (QIS), Escala de Beck y Escala de Satisfacción con el Apoyo Social (ESSS). Se realizó la prueba de chicuadrado, prueba exacta de Fisher, cálculo de Odds-Ratio (OR). Ambas pruebas estadísticas se realizaron utilizando el programa Statistical Package for Social Sciences (SPSS). En el estudio participaron 130 estudiantes universitarios, predominantemente mujeres $(81,5 \%)$, con edades comprendidas entre 21 y 25 años $(61,5 \%)$ y solteros $(78,5 \%)$. Del total de la muestra, $28(21,53 \%)$ tenían riesgo de tener ideación suicida. Se enumeró que los estudiantes que viven en otra ciudad tienen 1,52 veces más probabilidades de tener ideación suicida (IC $=0,65-3,55$ ), que tienen un ingreso mensual menor o igual al salario mínimo aumenta en 2 veces las posibilidades de tener ideación suicida. (IC: 0,740,53) y afirmar padecer algún trastorno mental o conductual aumenta en 1,40 veces las posibilidades de ideación suicida (IC $=0,60$ a 3,25). Conocer las características mentales de los estudiantes universitarios permite planificar intervenciones conjuntas en la comunidad académica y desarrollar estrategias de prevención y promoción, reduciendo la ideación suicida en este público.
\end{abstract}

Palabras clave: Ideación suicida; Estudiantes; Salud del estudiante; Universidades.

\title{
1. Introdução
}

Os transtornos mentais são condições clínicas caracterizadas por alterações emocionais e comportamentais relacionadas à angústia pessoal ou à deterioração do funcionamento psíquico, tendo efeitos deletérios, atingindo não somente o indivíduo, mas a família e a comunidade. Na atualidade as demandas sociais podem acarretar no indivíduo um aumento do sofrimento psíquico, fato que leva muitos a desenvolverem transtornos mentais e até mesmo a tentarem contra a própria vida (Carvalho et al., 2017).

As experiências negativas da vida, podem comprometer o desenvolvimento emocional de alguns indivíduos, sobrecarregando-os e aumentando a tensão emocional, e essas experiências podem ser inúmeras e de causas aparentemente corriqueiras, levando a um tensionamento de um transtorno pré-existente, o que causa um desarranjo psíquico forte, que leva o indivíduo a idealizar o fim desse sofrimento (Muller et al., 2017).

Os transtornos mentais são importantes vulnerabilizadores para o suicídio, podendo ou não se associar com consumo excessivo de álcool e outras drogas, doenças crônicas, violência, mudanças repentinas e importantes na vida da pessoa, situação cultural e socioeconômica (Santos et al., 2017). O suicídio é considerado um fenômeno complexo e multicausal que repercute socialmente e se caracteriza como um ato com resultado letal. A ideação suicida é umato sem resultado fatal, no qual o indivíduo se lesiona ou apresenta pensamentos persistentes relacionados ao suicídio (Melo et al., 2019).

De acordo com a organização Panamericana de Saúde (OPAS), (2016) 800 mil pessoas morrem por suicídio ao ano, e em sua maioria acontecem em países de baixa renda, sendo a tentativa o fator de risco mais importante e é a segunda principal causa de morte entre pessoas de 15 a 29 anos, corroborando com estes dados a Organização Mundial de Saúde (OMS), relata que no Brasil para esta faixa etária é a $3^{\mathrm{a}}$ causa de morte.

Segundo o DATASUS, no ano de 2019, em Minas Gerais, 1732 pessoas morreram por lesões autoprovocadas voluntariamente, sendo que mais de 7\% tinham 12 ou mais anos de estudo, e que destas 1732 pessoas 60\% (1036) se encontravam na faixa etária entre 20 a 49 anos, e dentre esses 1036, mais de $12 \%$ (125) apresentavam essa taxa de escolaridade.

Com compreensão destes dados Nogueira; Barros; Sequeira (2017), descrevem que a qualidade da saúde mental de jovens que não são universitários é superior quando comparados a jovens universitários da mesma idade. O ingresso na universidade ao mesmo tempo que se configura como um momento de satisfação por ser uma possibilidade de concretização profissional, pode também corresponder a um período de grandes mudanças e cheio de desafios. Sendo assim, os jovens universitários, se sentem pressionados devido vários dilemas relacionados ao amadurecimento e ao futuro, que estão 
associados com o desamparo social e que funcionam como um gatilho para agravar transtornos mentais (Akca; Yuncu; Aydin, 2018; Ribeiro et al., 2018).

Neste contexto, é importante dar mais atenção para ideação suicida, principalmente entre os jovens (Ribeiro; Moreira, 2018), pois sua progressão, aliada por meio de informações à cerca do planejamento claro e intencionalidade da pessoa, podem sinalizar efetivação do suicídio nas próximas horas ou dias (Del-Bem et al., 2017).

Portanto, entende-se que a ideação suicida é um problema recorrente na vida das pessoas, em especial da população jovem e universitária, que se encontra permeada por fatores sociais que podem influenciar nas tomadas de decisões. Assim, analisar as motivações e os fatores que se associam com a ideação suicida nos universitários é desejado, pois estes são acompanhados por responsabilidades e transformações na vida acadêmica, sendo importante buscar respostas frente aos desafios propostos pela ocorrência da ideação suicida, nas vítimas e as consequências sociais geradas. Desta forma, o objetivo deste estudo foi analisar se há ideações suicidas nos jovens universitários e quais as motivações, bem como descrever o perfilsociodemográfico e econômico dos estudantes.

\section{Metodologia}

Trata-se de um estudo do tipo transversal que segundo Hulley et al., 2015, consiste em uma ferramenta para a descrição de características da população afim de auxiliar na ação e no planejamento em saúde. Com abordagem quantitativa que utiliza a linguagem matemática para descrever as causas de um fenômeno (Teixeira, 2011). Desenvolvido na Universidade do Estado de Minas Gerais - UEMG, Unidade Divinópolis no ano de 2020. A amostra foi composta por 146 alunos dentro dos 2.294 matriculados em 2020, nos diferentes cursos da UEMG.

Os critérios de inclusão foram: universitários que cursam a partir do terceiro período por terem maior vivência na universidade, ambos os sexos, regularmente matriculados e idade superior a 18 anos. Como critérios de exclusão foram: universitários menores de 18 anos, alunos que cursavam o primeiro e segundo período e alunos que preencheram o questionário mais de uma vez. Dentre os 146 respondentes, 16 questionários não foram utilizados, devido aos critérios de inclusão e exclusão, sendo o final da amostra 130 universitários. A coleta de dados ocorreu por meio da plataforma de formulários GoogleForms, no período de outubro a dezembro de 2020.

Foi utilizado quatro questionários que possibilitaram identificar a caracterização das variáveis sociodemográficas, econômicas, acadêmicas, eventos da vida e o histórico de tentativa de suicídio do universitário, sendo eles: Questionário Sociodemográfico, Suicide Ideation Questionnaire (QIS), Escala de Desesperança de Beck e a Escala de Satisfação com o Suporte Social.

Os dados sobre ideação suicida foram obtidos a partir do QIS, versão portuguesa do Suicide Ideation Questionnaire, de Reynolds (1998), traduzida e adaptada para a populaçãoportuguesa por Ferreira e Castela (1999). Esse questionário tem como objetivo analisar a ocorrência de ideação suicida em adolescentes e adultos, constituído por 30 itens e com respostas estruturadas numa escala de Likert com 6 valores: 0 - nunca tive este pensamento; 1 - pensei nisto, mas não no último mês; 2 - cerca de uma vez por mês; 3 - duas vezes por mês; 4 - cerca de uma vez por semana; 5 - duas vezes por semana; 6 - quase todos os dias. O valor daescala é obtido somando as pontuações dos 30 itens, e varia entre 0 e 180 pontos. Quanto maiora pontuação, maior a ideação suicida. De acordo com Reynolds (1988), a pontuação no QIS superior ou igual a 41 pontos pode ser indicativa da presença de psicopatologia e de um potencial risco de suicídio (Reynolds, 1998; Vasconcelos-Raposo et al., 2016).

Já a Escala de Beck, avalia a extensão das expectativas negativas a respeito do futuro imediato e remoto. Possui 20 afirmações que envolvem cognições sobre desesperança, que o escore total pode variar de 0 a 20 permitindo a classificação 
da desesperança em níveis. Os pontos de corte e níveis são: de 0 a 4, nível mínimo; de 5 a 8, nível leve; de 9 a 13, nível moderado e entre 14 e 20, nível grave (Oliveira, 2006).

A Escala de Satisfação com o Suporte Social (ESSS), avalia a satisfação com o suporte social existente, como a satisfação com amigos e família, intimidade e atividades sociais. Constitui-se de 15 itens, e a pontuação da escala é dividida em três partes: alto suporte social (de 51 a 75 pontos); médio suporte social (de 26 a 50 pontos) e baixo suporte social (até 25 pontos) (Ribeiro, 1999).

Para verificar se os questionários eram eficazes para contemplar os objetivos deste estudo, foi realizado um teste piloto com sete pessoas escolhidas de forma aleatória, anteriormente ao início da coleta de dados com os participantes previstos no estudo. As informações oriundas desta etapa não compuseram o banco de dados para análise.

Para validação dos construtos foi utilizado o teste de qui-quadrado de aderência para verificar se há semelhança do estudo com os dados da literatura, com base no questionário utilizado (QIS), apresentou semelhança satisfatória e para análise das variáveis qualitativas foiutilizado o Teste de Fisher.

Os dados foram armazenados em planilha eletrônica no formato Microsoft Excel versão 2016, posteriormente, foi realizado a análise descritiva dos dados. Logo após, realizou o teste, Kolmogorov-Smirnov para avaliar a distribuição normal que utiliza as hipóteses, para identificar visualmente as variáveis envolvidas no estudo que se relacionam entre si. Os testes estatísticos foram realizados através do programa Statistical Package for Social Sciences (SPSS) versão 20.0 considerando o nível de significância de 5\% (IBM, 2017).

No formulário, os participantes foram esclarecidos quanto ao objetivo, método do estudo e a assinar o Termo de Consentimento Livre e Esclarecido (TCLE). O sigilo das respostas foi assegurado pelo anonimato e o preenchimento do formulário não foi obrigatório. Essa estratégia garantiu confidencialidade e privacidade conforme as diretrizes éticas e legais da Resolução no . 510/2016. O estudo recebeu aprovação do Comitê de Ética em Pesquisa (CEP) da UEMG (Parecer 4.284.852), em 18 de setembro de 2020.

\section{Resultados}

Os participantes do estudo apresentaram idade entre 19 e 63 anos. Predominaram a faixaetária entre 21 a 25 anos 80 (61,5\%), do sexo feminino $106(81,5 \%)$, solteiros 102 (78,5\%), com renda mensal $\leq$ a 1 salário mínimo 88 (67,7\%), sem vínculo empregatício 81 (62,3\%), residindo na cidade da universidade 80 (61,5\%), consomem bebidas alcoólicas 93 (71,5\%) e $8(6,2 \%)$ fazem uso de tabaco (Tabela 1$)$. 
Tabela 1 - Participantes do estudo agrupados por sexo, idade, estado civil, local onde reside,curso em que está matriculado, vínculo empregatício, renda, consumo de álcool e tabaco.

\begin{tabular}{|c|c|c|}
\hline & $\mathbf{N}$ & $\%$ \\
\hline \multicolumn{3}{|l|}{ Sexo } \\
\hline Feminino & 106 & 81,5 \\
\hline Masculino & 24 & 18,5 \\
\hline Total & 130 & 100 \\
\hline \multicolumn{3}{|l|}{ Idade } \\
\hline 18 a 20 & 16 & 12,3 \\
\hline 21 a 25 & 80 & 61,5 \\
\hline 26 a 30 & 6 & 4,61 \\
\hline 31 a 35 & 11 & 8,4 \\
\hline 36 a 40 & 5 & 3,8 \\
\hline Acima de 40 & 8 & 6,15 \\
\hline Total & 130 & 100 \\
\hline \multicolumn{3}{|l|}{ Estado Civil } \\
\hline Solteiro & 102 & 78,5 \\
\hline Casado & 15 & 11,5 \\
\hline Total & 130 & 100 \\
\hline \multicolumn{3}{|l|}{ Residência } \\
\hline Divinópolis & 80 & 61,5 \\
\hline Outra cidade & 50 & 38,4 \\
\hline Total & 130 & 100 \\
\hline \multicolumn{3}{|l|}{ Curso } \\
\hline Enfermagem & 57 & 43,8 \\
\hline Educação Física & 12 & 9,2 \\
\hline Pedagogia & 12 & 9,2 \\
\hline Fisioterapia & 11 & 8,5 \\
\hline Outros & 38 & 29,3 \\
\hline Total & 130 & 100 \\
\hline \multicolumn{3}{|c|}{ Vínculo empregatício } \\
\hline Sim & 49 & 37,6 \\
\hline Não & 81 & 62,3 \\
\hline Total & 130 & 100 \\
\hline \multicolumn{3}{|l|}{ Renda } \\
\hline$\leq 1$ salário mínimo & 88 & 67,7 \\
\hline$>1$ salário mínimo & 42 & 32,3 \\
\hline Total & 130 & 100 \\
\hline \multicolumn{3}{|l|}{ Consome Álcool } \\
\hline Sim & 93 & 71,5 \\
\hline Não & 37 & 28,5 \\
\hline Total & 130 & 100 \\
\hline \multicolumn{3}{|l|}{ Consome Tabaco } \\
\hline Sim & 8 & 6,2 \\
\hline Não & 122 & 93,8 \\
\hline Total & 130 & 100 \\
\hline
\end{tabular}

Fonte: Dados da pesquisa.

Na tabela 2, verifica-se que 61 (46,9\%) afirmaram que sofrem de algum transtorno mental e/ou comportamental, 31 $(23,8 \%)$ possuem algum familiar que tentou terminar com a própria vida, 18 (13,8\%) possuem algum familiar que cometeu autoextermínio e $76(58,4 \%)$ afirmaram terem vivenciado algum tipo de acontecimento adverso nos últimos anos. 
Tabela 2 - Saúde e problemas mentais relatados pelos participantes do estudo.

\begin{tabular}{lcc}
\hline \multicolumn{1}{c}{$\mathbf{N}$} & $\%$ \\
\hline \multicolumn{2}{l}{ Sofre de algum transtorno } & mental/comportamental \\
Sim & 61 & 46,9 \\
Não & 69 & 53,0 \\
Total & 130 & 100 \\
\hline \multicolumn{2}{l}{ Possui familiar que tentou terminar com a própria } \\
vida & \\
Sim & 31 & 23,8 \\
Não & 99 & 76,1 \\
Total & 130 & 100 \\
\hline Possui familiar que cometeu autoextermínio \\
Sim & 18 & 13,8 \\
Não & 112 & 86,1 \\
Total & 130 & 100 \\
\hline Vivenciou algum acontecimento adverso nos últimos \\
anos & & \\
Sim & 76 & 58,4 \\
Não & 54 & 41,5 \\
Total & 130 & 100 \\
\hline
\end{tabular}

Fonte: Dados da pesquisa.

Da amostra total que responderam o QIS que objetiva analisar a ocorrência de ideação suicida 28 (21,53\%) apresentaram pontuação maior que 41, sendo indicativo de ter ideação suicida. Já com a aplicação da Escala de Beck, com a intenção de avaliar a extensão das expectativas negativas a respeito do futuro, a maioria dos estudantes apresentaram nível moderado de desesperança 98 (75,38\%), seguido de 27 (20,76\%) nível leve e 5 (3,84\%) nível grave. E na ESSS que avalia a satisfação com os amigos e família, intimidade e atividades sociais, 67 (51,53\%) declararam nível alto de satisfação com o suporte social; seguido de 57 (43,84\%) nível médio e 6 (4,61\%) nível baixo.

Observou-se por meio do QIS que do total de universitários entrevistados 28 (21,53\%) apresentaram ideação suicida, dentre estes, 29,2\% eram do sexo masculino, 19,1\% consomem bebida alcoólica, 12,5\% fazem uso de tabaco, 19,4\% possuem familiares que tentaram pôr fim a própria vida e 11,1\% possuem familiares que terminaram com a própria vida (Tabela 3). 
Tabela 3 - Associação entre a possibilidade de ideação suicida e fatores sociais e econômicos dos participantes do estudo.

\begin{tabular}{|c|c|c|c|c|c|c|c|}
\hline \multicolumn{4}{|l|}{ Ideação Suicida } & & \multirow[t]{3}{*}{ OR } & \multirow[t]{3}{*}{ IC } & \multirow[t]{3}{*}{ p-valor } \\
\hline & \multicolumn{2}{|l|}{ Sim } & \multicolumn{2}{|c|}{ Não } & & & \\
\hline & $\mathbf{N}$ & $\%$ & $\mathbf{N}$ & $\%$ & & & \\
\hline \multicolumn{5}{|l|}{ Sexo } & 1,667 & $0,612-4,537$ & 0,314 \\
\hline Feminino & 21 & 19,8 & 85 & 83,3 & & & \\
\hline Masculino & 7 & 29,2 & 17 & 16,7 & & & \\
\hline \multicolumn{5}{|l|}{ Residência } & 1,523 & $0,654-3,545$ & 0,328 \\
\hline Divinópolis & 15 & 18,8 & 65 & 81,2 & & & \\
\hline Outra cidade & 13 & 26,0 & 37 & 74,0 & & & \\
\hline \multicolumn{5}{|l|}{ Renda } & 2,000 & $0,743-0,5382$ & 0,165 \\
\hline$\leq 1$ salário mínimo & 22 & 25,0 & 66 & 75,0 & & & \\
\hline > 1 salário mínimo & 6 & 14,3 & 36 & 85,7 & & & \\
\hline \multicolumn{5}{|l|}{ Transtornos Mentais } & 1,405 & $0,607-3,250$ & 0,426 \\
\hline Sim & 15 & 24,6 & 46 & 74,5 & & & \\
\hline Não & 13 & 18,8 & 56 & 81,2 & & & \\
\hline \multicolumn{5}{|l|}{ Uso de álcool } & 1,159 & $0,860-1,562$ & 0,284 \\
\hline Sim & 18 & 19,1 & 76 & 80,9 & & & \\
\hline Não & 10 & 27,8 & 26 & 72,2 & & & \\
\hline \multicolumn{5}{|l|}{ Uso de tabaco } & 1,922 & $0,247-14,973$ & 0,453 \\
\hline Sim & 1 & 12,5 & 7 & 87,5 & & & \\
\hline Não & 27 & 22,1 & 95 & 77,9 & & & \\
\hline \multicolumn{5}{|c|}{ Familiares que tentaram pôr fim a própria vida } & 1,144 & $0,521-2,512$ & 0,735 \\
\hline Sim & 6 & 19,4 & 25 & & & 80,6 & \\
\hline Não & 22 & 22,2 & 77 & & & 77,8 & \\
\hline \multicolumn{5}{|c|}{ Familiares que terminaram com a própria vida } & 2,196 & $0,537-8,988$ & 0,359 \\
\hline Sim & 2 & 11,1 & 16 & & & 88,9 & \\
\hline Não & 26 & 23,2 & 86 & & & 76,8 & \\
\hline
\end{tabular}

Fonte: Dados da pesquisa.

Entre os universitários com presença de ideação suicida, 26,0\% relataram não residir na mesma cidade da universidade, $25,0 \%$ recebem menor ou igual a um salário-mínimo e 24,6\% alegaram sofrer algum transtorno mental ou comportamental.

De acordo com os dados apresentados na tabela 3, os universitários que residem em outra cidade, possuem 1,52 vezes mais chances de apresentarem ideação suicida $(\mathrm{IC}=0,65-3,55)$. E os universitários que alegaram possuir renda mensal menor ou igual a um salário-mínimo aumenta em 2 vezes as chances de ter ideação suicida (IC: 0,74-0,53). E que alegaram sofrer de algum transtorno mental ou comportamental apresentam 1,40 vezes mais chances de ideação suicida $($ IC $=0,60-3,25)($ Tabela 3).

Identificou-se que há correlação negativa entre o escore do QIS com o ESSS (p-valor=0,000), e entre o escore da Escala de Beck e o ESSS (p-valor=0,000), ou seja, quanto maior o escore do QIS e da Escala de Beck, menor a satisfação com o suporte social existente.

\section{Discussão}

Os resultados das condições sociodemográficas e econômicas obtidas nesse estudo vão ao encontro de outras pesquisas envolvendo estudantes universitários que apresentam ideações suicidas. Em relação à faixa etária é notável a predominância de idades entre 18 a 25 anos e sexo feminino, nas pesquisas realizadas na Universidade Federal de Mato Grosso e em uma instituição pública de ensino superior do Estado do Piauí (Santos et al., 2017; Santos et al., 2019). A população adulta jovem é mais acometida, sendo evidenciado na literatura, geralmente nos primeiros dois anos da graduação, que existe um aumento na prevalência do comportamento suicida em diferentes países (Santos et al., 2020; Silva et al., 2017). 
Em relação ao estado civil, 102 (78,5\%) se declararam solteiros, o que corrobora com o estudo realizado em um curso da saúde de um Centro Universitário no Ceará em que 92,0\% dos estudantes eram solteiros (Leão et al., 2018). Além disso, outros estudos, identificaram uma possível associação entre o jovem universitário morar sozinho e/ou longe da família com a ideação suicida (Silva et al., 2017; Pereira \& Cardoso, 2015). O que também pode ser observado nos dados aqui encontrados, onde 13 (26,0\%) dos participantes que moram em outras cidades apresentaram risco para ideação suicida.

$\mathrm{O}$ fato do distanciamento social da rede familiar e dos amigos, pode fazer surgir um sentimento de não integração social e solidão, resultando na criação de condições propensas ao surgimento de ideação (Leão et al., 2018). Além disso, outras situações que podem ser evidenciadas são as dificuldades financeiras, aumento do consumo de álcool, a existência de sintomas depressivos e falta do apoio social (Vêncio et al., 2019; Azeredo \& Afonso, 2016). Deste modo, considera-se que jovens que residem sozinhos e possuem estado civil solteiro, tem maior prevalência de adoecimento mental, sendo possivelmente ocasionado pela ausência de companhia para dividir e compartilhar experiências vivenciadas no dia a dia.

Outro ponto evidenciado nos dados coletados, é a relação de ideação suicida com baixa condição socioeconômica, 22 $(25,0 \%)$ dos entrevistados que possuem renda menor ou igual a um salário mínimo apresentaram risco de ideação suicida. Dado que corrobora aos achados da literatura, em que o desemprego e o baixo nível socioeconômico são apontados como riscos de tentativa de suicídio, principalmente, em indivíduos com histórico de tentativa de suicídio (Félix, 2016; Santos, 2020).

Os indivíduos que convivem com sintomas depressivos, exteriorizam frequentemente o desejo em morrer e/ou se matar, chegando à dedução que cometer o suicídio é a solução (Aquino, 2019; Cybulski \& Mansani, 2017). Confirmando essa informação, neste estudo 15 (24,6\%) dos participantes que possuem algum transtorno mental apresentam a possibilidade de ter ideação suicida. Assim, o suicídio surge como a única saída existente diante de um momento conflituoso e de expectativas negativas para o futuro. Quando o indivíduo apresenta sintomas depressivos podem ocorrer falta de sentido na vida e sensação de impotência, que o aparecimento dessa sensação fortifica esse contexto predispondo o estudante à ideação suicida.

$\mathrm{O}$ ambiente universitário possibilita novas descobertas e vínculos afetivos e relacionais, bem como participação em círculos sociais por meio de festas. Para ser aceito neste meio, écomum que os jovens adotem posturas vulneráveis, dentre elas o uso de álcool, tabaco e outrasdrogas (Veloso et al., 2019; Akca, 2018). Os achados desse estudo relacionadas ao uso de álcool apontam que $18(19,1 \%)$ das pessoas que consomem, apresentam ideação suicida, mesmo que mínimas, existe uma associação entre o uso dessas substâncias e a presença de ideações suicidas.

A prevalência do uso do tabaco na população observada foi pequena, em ambos os sexos. Assim como pesquisa realizada com estudantes da área da saúde no Nordeste (Leão et al., 2018). Entretanto, em trabalho realizado no Piauí com estudantes da área da saúde a realidade foi diferente, pois, o estudo evidenciou que existe grande relação entre o uso desta substância e a presença de ideação suicida (Veloso et al., 2019). A percepção dos autores em relação a este resultado, defende que pode ter acontecido um viés de informação, onde os participantes não entenderam que tabaco é similar a cigarro (tipo industrial ou de palha) ou relacionaram a substância com algum tipo de droga ilícita. Pois, percebe-se que há muitos estudantes que fumam nos ambientes da instituição.

O histórico de tentativas de suicídio possui valor preditivo na avaliação do risco de suicídio, uma vez que a reincidência do comportamento suicida normalmente é observada por meio de pensamentos recorrentes de morte (Veloso et al., 2019). E apesar de neste estudo essa relação não ter sido evidenciada, em outros estudos realizados nos estados mineiro e mato-grossense em que 9,5\% e 9\% dos universitários, respectivamente, reportaram histórico de tentativa de suicídio (Santos et al., 2017; Lappann Botti et al., 2016). Sendo assim, a identificação e o acompanhamento de universitários com esse tipo de histórico deve ser priorizado no espaço da universidade, de modo que direcione atenção e condutas de professores, servidores e demais alunos, com vistas ao fortalecimento de vínculos e da escuta qualificada. 
Com base nessas evidências, a instituição de ensino deve propor estratégias para acolhimento aos estudantes com ideação suicida, elaborando grupos e salas de apoio pelos estudantes e docentes de Psicologia. Além disso, os demais universitários dos cursos de saúde como a Enfermagem, Fisioterapia e Educação Física, poderiam elaborar programas de saúde, atividades coletivas no campus da universidade, permitindo uma escuta qualificada e o desenvolvimento de ações que estimulem os alunos a socializarem e a trabalharem seus problemas de uma forma saudável.

A variável renda (menor ou igual um salário-mínimo) foi identificada como um fator de chance para ideação, desse modo, a implementação de bolsas de auxílio e incentivo ao aluno a participar de projetos com bolsas oferecidas pelos programas vinculados a universidade favorece uma melhoria na renda econômica do estudante. Desta maneira, a universidade pode tornar-se um fator protetivo para novos casos de ideação suicida.

O presente estudo apresenta como limitações a existência da pandemia pelo Coronavírus(Covid-19) que interferiu na realização da pesquisa presencialmente, além disso, não foi possível identificar quais alunos negaram preencher o formulário devido a temática abordada.

\section{Conclusão}

O momento da graduação é marcado por grandes desafios, que muitas vezes pode-se caracterizar como um momento de dificuldade para o universitário, pois além da vida acadêmica o indivíduo precisa aprender a lidar com dificuldades relacionadas ao desempenho pessoal, social, que demanda maturidade e autonomia. Entretanto, quando há dificuldades relacionadas a essas demandas pode se observar sentimentos de preocupação, incerteza, tristeza, fracasso e não familiaridade com a própria vida, que podem progredir para ideações suicidas.

Sendo assim, é importante salientar que são inúmeras as consequências do suicídio, podendo envolver prejuízos emocionais aos familiares e amigos, sociais e também econômicos, principalmente em faixas etárias mais jovens em que o impacto pode ser mais acentuado.

Portanto, conhecer as características mentais dos universitários, possibilita o planejamento de intervenções através de ações conjuntas na comunidade acadêmica e civil, favorecendo assim um processo de discussão e identificação das necessidades individuais dos universitários. Disponibilizando um suporte de apoio psicológico, físico e social, com estratégias voltadas para a prevenção e promoção da saúde mental, no intuito de diminuir a ideação suicida nesse público.

Ressaltamos que esta pesquisa não esgota o tema ou os sujeitos da pesquisa, observamos necessidade de novos estudos sobre a ideação suicida em jovens universitários, bem como pesquisas que abordem maneiras de lidar com o adoecimento psíquico dentro da universidade evitando assim desfechos trágicos.

\section{Referências}

Aquino, D. R., Cardoso, R. A. \& Pinho, L. (2019). Sintomas de depressão nas universidades médicas. Boletim-Academia Paulista de Psicologia, 39(96), 8195 .

Akca, S. O., Yuncu, O. \& Aydin, Z. (2018). Mental status and suicide probability of young people:A cross - sectional study. Revista da Associação Médica Brasileira, 64(1), 32-40.

Azeredo, Z. D. A. S., \& Afonso, M. A. N. (2016). Solidão na perspectiva do idoso. Revista Brasileira de Geriatria e Gerontologia, $19,313-324$.

Brasil. (2019). Ministério da Saúde. Secretaria de Vigilância em Saúde/CGIAE - Sistema de Informações sobre Mortalidade. Banco de dados do Sistema Único de Saúde-DATASUS. http://tabnet.datasus.gov.br/cgi/tabcgi.exe?sim/cnv/ext10mg.def>

Brasil. (2017). Ministério da Saúde; Secretaria de Atenção à Saúde (Org.). Prevenção do suicídio: sinais para saber e agir. São Paulo: Universidade Estadual de Campinas. <https://www.saude.gov.br/saude-de-a-z/suicidio>

Brasil. (2016). Resolução n ${ }^{\circ}$ 510, de 7 de abril de 2016. Dispõe sobre as normas aplicáveis a pesquisas em Ciências Humanas e Sociais. Diário Oficial da União. http://conselho.saude.gov.br/resolucoes/2016/Reso510.pdf 
Botti, N. C. L., Monteiro, A. M. C., Benjamin, M. L. N. \& Queiroz, L. C. (2016). Depressão, Uso de drogas, Ideação e Tentativa de Suicidio entre estudantes de enfermagem. Journal of Nursing UFPE/Revista de Enfermagem UFPE, 10(7).

Carvalho, K., Jardim, R., Barbosa, S. \& Luiz, G. M. (2017). Suicídio: o último estágio da dor humana (TCC-Psicologia).

Cybulski, C. A. \& Mansani, F. P. (2017). Análise da depressão, dos fatores de risco para sintomasdepressivos e do uso de antidepressivos entre acadêmicos do curso de medicina da Universidade Estadual de Ponta Grossa. Revista brasileira de educação médica, 41(1), 92-101.

Del-Ben, C. M., Sponholz- Junior, A., Mantovani, C., Faleiros, M. C. M., Oliveira, Guapo, V. G., \& Marques, J. M. A. (2017). Emergências Psiquiátricas: manejo de agitação psicomotora e avaliaçãode risco suicida. Medicina (Ribeirão Preto, Online.), 50(1), 98-112.

Ferreira, J. A. \& Castela, M. C. (1999). Questionário de Ideação Suicida (Q.I.S). Braga: Sistemas Humanos e Organizacionais.

Félix, T. A., Oliveira, E. N., Lopes, M. V. O., Parente, J. R. F., Dias, M. S. A. \& Moreira, R. M. M. (2016). Fatores de risco para tentativa de suicídio: produção de conhecimento no Brasil. Revista Contexto \& Saúde, 16(31), 173-185.

Hulley, S. B., Cummings, S. R., Browner, W. S., Grady, D. G., \& Newman, T. B. (2015). Delineando a pesquisa clínica-4. Artmed Editora.

Ibm, I. I. (2017). Statistics for Windows, Versão 25.0. Armonk, IBM Corporation.

Leão, A. M., Gomes, I. P., Ferreira, M. J. M. \& Cavalcanti, L. P. G. (2018). Prevalência e fatores associados à depressão e ansiedade entre estudantes universitários da área da saúde de um grande centro urbano do Nordeste do Brasil. Revista brasileira de educação médica, 42(4), 55-65.

Melo, M. M., Nespoli, B. R., Santana, R. S., Fontes, F. L. L., Junior, J. G. A. L., \& Carvalho, A. D. T. (2019). Percepções da equipe multiprofissional de um Centro de Atenção Psicossocial sobre as motivações que levam o sujeito ao comportamento suicida. Revista Eletrônica Acervo Saúde, (24), 529.

Müller, S. A., Pereira, G. S. \& Zanon, R. B. (2017). Estratégias de prevenção e pósvenção do suicídio: Estudo com profissionais de um Centro de Atenção Psicossocial. Revista de Psicologia da IMED, Passo Fundo, 9(2), 6-23.

Nogueira, M. J., Barros, L., Sequeira, C. (2017). A saúde mental em estudantes do ensino superior. Relação com o género, nível socioeconómico e os comportamentos de saúde. Revista Portuguesa de Enfermagem de Saúde Mental, (5), 51-56.

Oliveira, K. L., Santos, A. A. A., Cruvinel, M. \& Néri, A. L. (2006). Relação entre ansiedade, depressão e desesperança entre grupos de idosos. Psicologia em Estudo, 11(2), 351-359.

Organização Pan-americana de Saúde. (2021). Suicídio. Brasília: Autor. https://www.paho.org/pt/topicos/suicidio.

Pereira, A. G. \& Cardoso, F.S. (2015). Ideação Suicida na População Universitária: Uma Revisão de Literatura. Revista E-Psi, 5(2), 16-34.

Ribeiro, J. L. P. (1999). Escala de satisfação com o suporte social (ESSS). Análise psicológica, 17(3), 547-558.

Ribeiro, J. M. \& Moreira, M. R. (2018). Uma abordagem sobre o suicídio de adolescentes e jovensno Brasil. Ciência \& Saúde Coletiva, 23(9), 2821-2834

Ribeiro, N. M., Castro, S. S., Scatena, L. M. \& Haas, V. J. (2018). Análise da Tendência Temporal do Suicídio e de Sistemas de Informações em Saúde em Relação às Tentativas de Suicídio. Texto Contexto Enfermagem, 27(2).

Reynolds, W. M. (1998). Suicidal ideation questionnaire: Professional manual. Psychological Assessment Resources.

Santos, A. G. G., Lima, D. S., Santos, F. C., Mehinako, M. N. \& Ferreira, L. D. S. (2020). Ideação Suicida entre Discentes: estudo da prevalência numa instituição privada de ensino superior em Belo Horizonte. Brazilian Journal of Development, 6(10), 81237-81267.

Santos, H. G. B., Marcon, S. R., Espinosa, M. M., Baptista, M. N. \& Paulo, P. M. C. (2017). Fatores associados à presença de ideação suicida entre universitários. Revista Latino-Americana de Enfermagem, (25).

Santos, H. G. B., Marcon, S., Espinosa, M. M. \& Baptista, M. N. (2019) Ideação suicida em estudantes universitários: um perfil sociodemográfico. Psicologia Argumento, 36(92), 237-253.

Santos, R. S., Alburquerque, M. C. S., Brêda, M. Z., Bastos, M. L. A., Silva, V. M. S. \& Tavares, N. V. S. (2017). Atuação do enfermeiro com a pessoa em situação de suicídio: análise reflexiva. Revista de enfermagem UFPE on line, 11(2) 742-748.

Silva, N. K. N., Carvalho, C. M. S., Magalhães, J. M., Carvalho Junior, J. A. M., Sousa, B. V. S., \& Moreira, W. C. (2017). Ações do enfermeiro na atenção básica para prevenção do suícidio. SMAD Revista Eletrônica Saúde Mental Álcool e Drogas (Edição em Português), 13(2), 71-77.

Teixeira, E. As três metodologias: acadêmica, das ciências e da pesquisa. Editora Vozes, 2011.

Vasconcelos-Raposo, J., Soares, A. R., Silva, F., Fernandes, M. G. \& Teixeira, C. M. (2016). Níveis de ideação suicida em jovens adultos. Estudos de Psicologia, 33(2)345-354.

Veloso, L. U. P., Lima, C. L. S., Sales, J. C. S., Monteiro, C. F. S., Gonçalves, A. M. S. \& Júnior, F. J. G. S. (2019). Ideação suicida em universitários da área da saúde: prevalência e fatores associados. Revista Gaúcha de Enfermagem, (40).

Vêncio, A. P. S., Sousa, N. G., Paulo, K. C. M., Mathias, E. F. \& Aguilar, R. R. (2019). Início da vida universitária versus desejo suícida/The beginning of university life versus suicidal desire. Brazilian Journal of Development, 5(11), 25019- 2503. 